\title{
A JAVA User Interface for the Virtual Human
}

\author{
R. C. Ward, D. J. Strickler, J. S. Tolliver, and C. E. Easterly \\ Oak Ridge National Laboratory* \\ P.O. Box 2008 \\ Oak Ridge, Tennessee 37831-6418
}

Paper for Presentation at
IEEE/BMES Conference

Atlanta, GA

October 13-16, 1999

\begin{abstract}
The submitted manuscript has been authored by a
contractor of the U.S. Government No. DE-AC-5-

96OR22464. Accordingly, the U.S. Government retains

a nonexclusive, royalty-free license to publish or

reproduce the published form of this contribution, or

allow others to do so, for U.S. Government purposes.
\end{abstract}

*Managed by the Lockheed Martin Energy Research Corp. for the U.S. Department of Energy under Contract No. DE-AC05-960R22464. 


\title{
A Java User Interface for the Virtual Human
}

\author{
R. C. Ward, D. J. Strickler, J. S. Tolliver, and C. E. Easterly \\ Oak Ridge National Laboratory \\ Oak Ridge, Tennessee \\ email: rwd@ornl.gov
}

\begin{abstract}
A human simulation environment, the Virtual Human (VH), is under development at the Oak Ridge National Laboratory (ORNL). Virtual Human connects threedimensional (3D) anatomical models of the body with dynamic physiological models to investigate a wide range of human biological and physical responses to stimuli. We have utilized the Java programming language to develop a flexible user interface to the $\mathrm{VH}$. The Java prototype interface has been designed to display dynamic results from selected physiological models, with user control of the initial model parameters and ability to steer the simulation as it is proceeding. Taking advantage of Java's Remote Method Invocation (RMI) features, the interface runs as a Java client that connects to a Java RMI server process running on a remote server machine. The RMI server can couple to physiological models written in Java, or in other programming languages, including $\mathrm{C}$ and FORTRAN. Future versions of the interface will be linked to $3 \mathrm{D}$ anatomical models of the human body to complete the development of the VH.
\end{abstract}

Introduction: Oak Ridge National Laboratory, in collaboration with other institutions, is developing the Virtual Human, a research/testing/training human simulation environment having an integrated system of physiological models, data, and advanced computational algorithms coupled with a 3D model of the anatomy. An important part of $\mathrm{VH}$ will be a user interface to the physiological and anatomical models. To provide an advanced, flexible, Web-based interface to $\mathrm{VH}$, we have developed a prototype using Java 2 which is accessible using a Web-browser such as Netscape Navigator 4.5 or Internet Explorer 5.

Methods: Using the World-Wide Web as an interface gives the greatest access to the VH. To take advantage of the Web, we have developed a Java applet that will access a library of physiological models and, utilizing user-supplied parameter modifications, execute a simulation under control of the interface. The Java applet was developed using Java 2 and takes advantage of the Swing graphical user interface classes. The applet uses RMI to connect to a remote server which in turn uses Java Native Interface (JNI) to connect to physiological models written in C or FORTRAN.

Results: The interface has proved to be very flexible and provides remote access to physiological simulations in tests conducted between ORNL and collaborators at Vanderbilt, East Tennessee State University, and the University of Tennessee
Medical Center in Knoxville. The users are able to view a simulation controlled by the initiators, who alone steer the simulations. We also tested the ability of remote collaborators to steer a simulation initiated at ORNL. Finally, we have integrated the Java user interface with 3D anatomical models rendered using Virtual Reality Modeling Language (VRML). This allows users to click on a selected organ in the 3D anatomy and display the appropriate physiological model and corresponding simulation results.

Discussion: Java proved to be a flexible language for the development of an interface for the $\mathrm{VH}$. A disadvantage of using Java is that Web browsers have not kept up to the current levels of the development of Java, requiring the use of the Java 2 plug-in to replace the Java virtual machine built into most browsers. The first time the applet is accessed, the user is required to download and install a 10-12 MB plug-in file if the plug-in has not already been installed on the user's system. Subsequent use of the applet will use the installed plug-in and not require downloading it again. This disadvantage will hopefully disappear as the browsers are brought up to the current level of Java. Advantages of using Java include the cross-platform capability and the object-oriented development paradigm, which allow us to quickly add new physiological models to the application. We continue to develop the interface, to improve the integration of the $3 \mathrm{D}$ anatomy and the physiological models using VRML, and to complete development of the capability to integrate models written in $\mathrm{C}$ or FORTRAN using metadata tables which define the model parameters, data, and results.

Conclusions: The Virtual Human simulation environment being developed at ORNL required a flexible, Web-based user interface for access to the anatomical and physiological models. Java proved to be a flexible tool for the development of this user interface. The Java interface is portable between Unix and Windows/NT platforms and it's object-oriented development paradigm makes it easy to extend to future physiological models. A test was conducted between collaborators on the Virtual Human Program which successfully proved the VH Java user interface will provide an excellent flexible, interactive means for accessing the Virtual Human. ORNL continues to be open to individuals and organizations interested in being collaborators on the multiyear Virtual Human effort. 